\title{
The Lair: a resource for exploratory analysis of published RNA-Seq data
}

Harold Pimentel ${ }^{1}$, Pascal Sturmfels², Nicolas Bray ${ }^{3}$, Páll Melsted ${ }^{4}$ and Lior Pachter ${ }^{1,5^{*}}$

\begin{abstract}
Increased emphasis on reproducibility of published research in the last few years has led to the large-scale archiving of sequencing data. While this data can, in theory, be used to reproduce results in papers, it is difficult to use in practice. We introduce a series of tools for processing and analyzing RNA-Seq data in the Sequence Read Archive, that together have allowed us to build an easily extendable resource for analysis of data underlying published papers. Our system makes the exploration of data easily accessible and usable without technical expertise. Our database and associated tools can be accessed at The Lair: http://pachterlab.github.io/lair.
\end{abstract}

Keywords: RNA-Seq, Sequence read archive, Exploratory data analysis, Shiny, Interactive visualization, Reanalysis, Reproducibility, Kallisto, Sleuth

\section{Background}

The Sequence Read Archive (SRA) is a public repository for sequencing data that has become an important archival resource for reads associated with published papers. The accumulation of large amounts of data in the SRA allows for meta-analyses that are possible only thanks to the centralized, open sharing of data by multiple investigators $[1,2]$. Reads in the SRA also allow, in principle, for the reproduction of results in publications [3]. However, the bioinformatics difficulties associated with processing and analyzing sequencing data [4] have limited the utility of the SRA and have made it prohibitive for most investigators to perform exploratory data analysis (EDA) on the data in the archive. The use of the SRA for EDA is especially difficult for RNA-Seq data. This is because even the most basic processing of RNA-Seq reads requires numerous decisions about appropriate software to use, complex choices about annotations, understanding of experimental design, and frequently, significant computational resources. As a result, workflows designed for operating on multiple datasets in the sequence read archive have mainly been restricted to the tasks of aligning

\footnotetext{
* Correspondence: Ipachter@math.berkeley.edu

'Department of Computer Science, University of California, Berkeley, 387 Soda Hall, Berkeley 94720, USA

${ }^{5}$ Departments of Mathematics and Molecular \& Cell Biology, University of California at Berkeley, Berkeley, CA 94720-3840, USA

Full list of author information is available at the end of the article
}

and quantifying reads [5]. Similarly, the Gene Expression Omnibus (GEO) requires an expression matrix to be uploaded with every sample, however these are static and frequently out of date, and fail to provide users with the complete analyses that they typically seek to explore.

We have recently developed a pair of tools called kallisto [6] and sleuth [7] for RNA-Seq analysis that address a number of the challenges associated with processing RNA-Seq data. The kallisto program circumvents the need for large alignment files, a convenience that reduces storage needs and increases speed, thus enabling the processing of large numbers of samples on modest computational resources. The program sleuth utilizes bootstraps output by kallisto for differential analysis, i.e. for determining how the expression of genes differ between samples, and provides a complete interactive and web-compatible solution for exploring and analyzing RNA-Seq data. This has allowed us to semi-automate the process of associating interactive Shiny-based [8] websites for EDA of RNA-Seq data. Shiny is a new web application framework for $\mathrm{R}$ that turns analyses into interactive web applications. 
The processing underlying the resource we have developed can easily be rerun, providing a scalable and updatable push-button system for the analysis of large numbers of datasets. Thus, we have been able to create a semi-automated system for analysis of archived RNA-Seq data that is much more informative than mere alignment and quantification and that opens up the analyses of published data.

\section{Construction and content}

The infrastructure for The Lair is based on Snakemake [9], a python-based workflow system. The system is organized as shown in Fig. 1 and consists of three main parts: (1) an initial processing of data to produce sleuth objects that are deployed in a Shiny database; (2) a Shiny server and database; and (3) a website that can be constructed automatically and that links to the Shiny server.

The specification for datasets is stored on GitHub at https://github.com/pachterlab/bears_analyses and is termed "bears analyses". Each dataset requires a config.json file that provides information about the dataset that will be used during its processing and deployment. For example, the config.json file for a recent paper on a HOXA1 knockdown transcriptome survey in human [10] is:
The config.json file specifies the species name for the RNA-Seq analysis, allowing Snakemake to automatically download and index the appropriate Ensembl [11] transcriptome for the analysis, the type of reads (single or paired), the design matrix and type of testing to perform, as well as the DOI of the paper and parameters for the kallisto processing. Along with the config.json file, a design matrix must be included which specifies the structure underlying the samples. The design matrix can be downloaded from the SRA but sometimes requires manual curation due to inconsistencies in SRA formatting. The entire workflow begins with only these two files, from which all the relevant information is extracted for processing.

The organization of The Lair allows for updating of all the analyses at the push of a button. This is useful in the case of updates to the component programs and the emphasis on speed of the constituent software allows for frequent updating. In fact, the current main bottleneck with The Lair is downloading the SRA data after which the entire workflow processes individual samples in minutes [6].

The website is a static page built using Jekyll [12] and Bootstrap v3.3.6 [13]. The Analyses section of the website contains a dynamically generated table of papers with corresponding live analyses. The table is powered by the JQuery plug-in DataTables [14] and features filtering

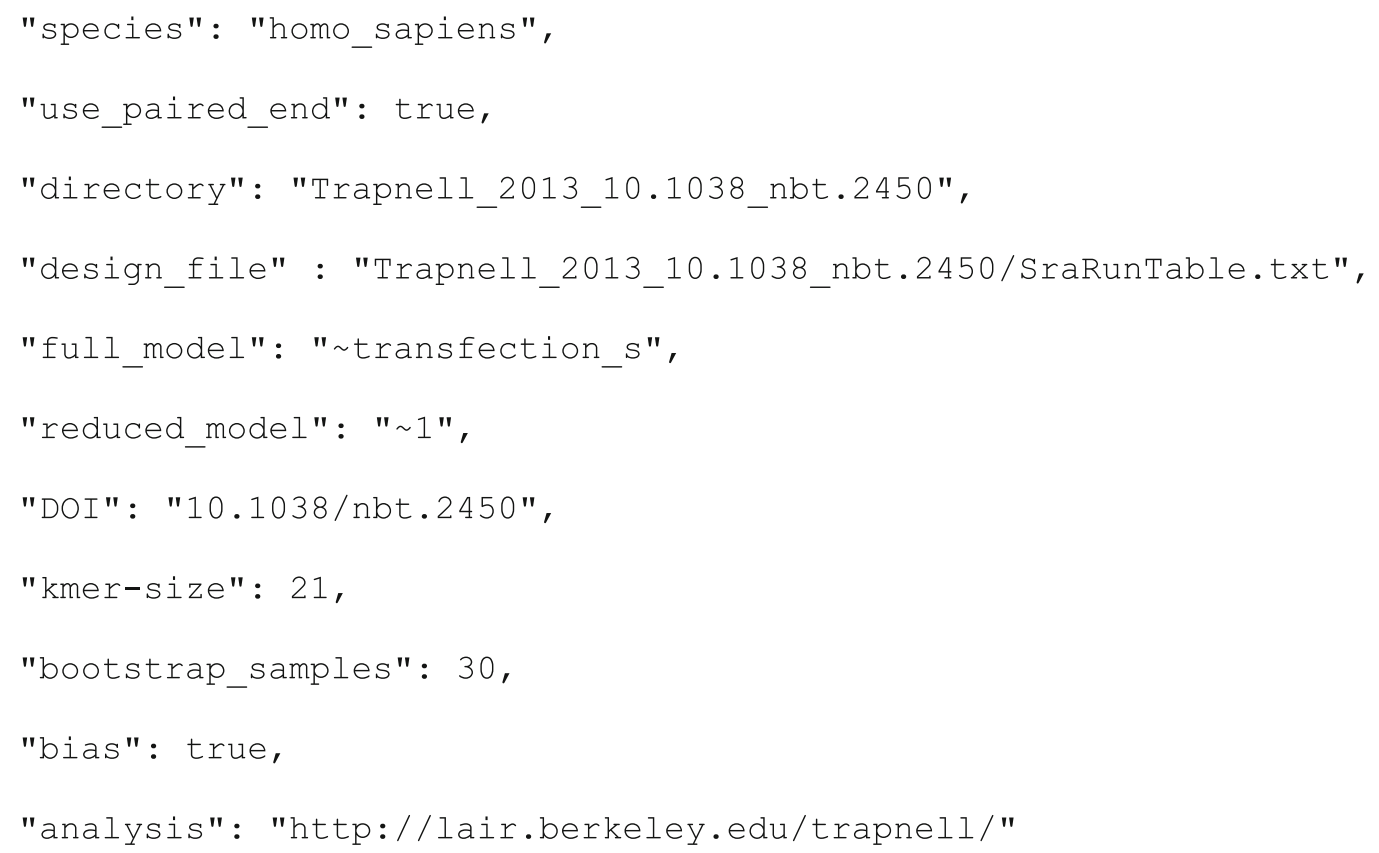




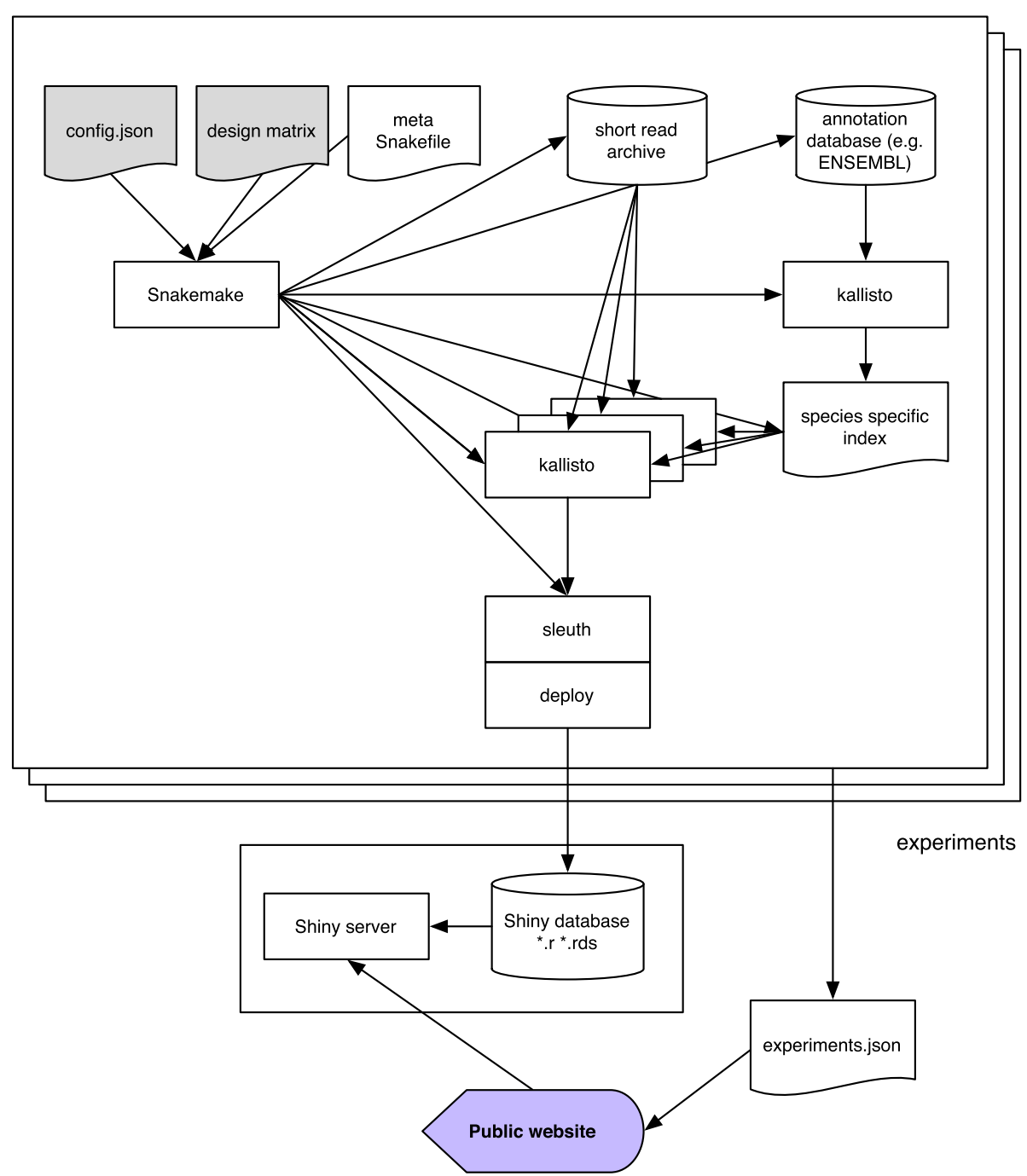

Fig. 1 Workflow of The Lair system for distributing analysis of sequence read archive data. The inputs to the system are sets of two files: config.json file that specifies parameters to be used during the processing of each experiment and a design matrix for each experiment that specifies its structure. A master Snakemake workflow organizes a series of computations starting with downloading of data to the sequence read archive and ending with deployment of a sleuth analyses to a Shiny server. Finally, a website generated from information in the config.json files links to objects in the Shiny server thus providing access to the processed experiments

and sorting by Authors, Title, Journal and Date. The title of each paper links to the original paper published in the stated journal. If the paper was published in print, the date given is the paper's print date; otherwise, the date is the paper's online publication date. The analysis button links to an in-browser analysis of the experiment, made possible by sleuth's efficient use of statistical bootstrapping and RStudio's Shiny plug-in; the analysis for each paper is generated automatically by the above build system.

The table is populated automatically by data from the bears analyses (https://github.com/pachterlab/bears_analyses) GitHub repository. This means that anyone can submit additional datasets for processing via GitHub pull requests which once accepted will become part of the website.

\section{Data handling}

There are two data bottlenecks in processing of sequence read archive RNA-Seq data: first, the downloading and storing of large read files, an issue we do not address in this paper but that can be ameliorated with compression schemes. Second, sleuth utilizes statistical bootstraps generated by kallisto as part of its differential transcript/gene analysis and these can be time consuming to transmit via the web. To make sleuth usable via The Lair, the code was refactored to pre-compute variance and quantile data that are sufficient and necessary statistics to generate the plots in the online visualization. The individual bootstraps estimates can then be discarded. This reduced the size of the analysis objects by orders of magnitude and allowed sleuth analyses to be shared online and loaded in standard web browsers. 


\section{The Snakemake workflow}

The Snakefile used to generate the analysis requires two input parameters: a json configuration file and a design matrix file. The configuration file has the following required parameters:

- species: species used in the experiment.

- used_paired_end: true if the experiment was paired-end, false otherwise.

- directory: the directory to put the results into.

- design_file: the name of the required design matrix file.

- full_model: formula which describes the full or alternative model used in differential analysis.

- reduced_model: formula which describes the reduced or null model.

- DOI: the digital object identifier of the publication.

The configuration file also accepts the following optional parameters:

- kmer-size: the k-mer length used to build the kallisto index (defaults to 31).

- bias: perform sequence-specific bias correction during quantification (defaults to True).
The design file must be a tsv file, one column of which is titled 'run' or 'Run_s' and contains the SRR accessions for each run in the experiment. Furthermore, the full_model and reduced_model in the config file must match column names in the design file for the differential analysis to work correctly.

The build system checks whether the FASTA reference file for the input species is already locally accessible. If not, it downloads it to the FASTA file from Ensembl. It then uses this FASTA file to build a kallisto index given the input k-mer size if the index does not already exist, and makes this index locally accessible.

Then the build system uses the 'run' or 'Run_s' column of the design matrix to download the raw SRA data and quantifies the raw data using kallisto and the index for the specified species. Once kallisto finishes quantification, sleuth is run with the likelihood ratio test using the specified full_model and reduced_model parameters. The build system then deploys the resulting sleuth analysis onto the server, where it is available to explore online.

\section{Utility and discussion}

To demonstrate the utility of The Lair we examined the results from our analysis of the Trapnell et al. [10] data.

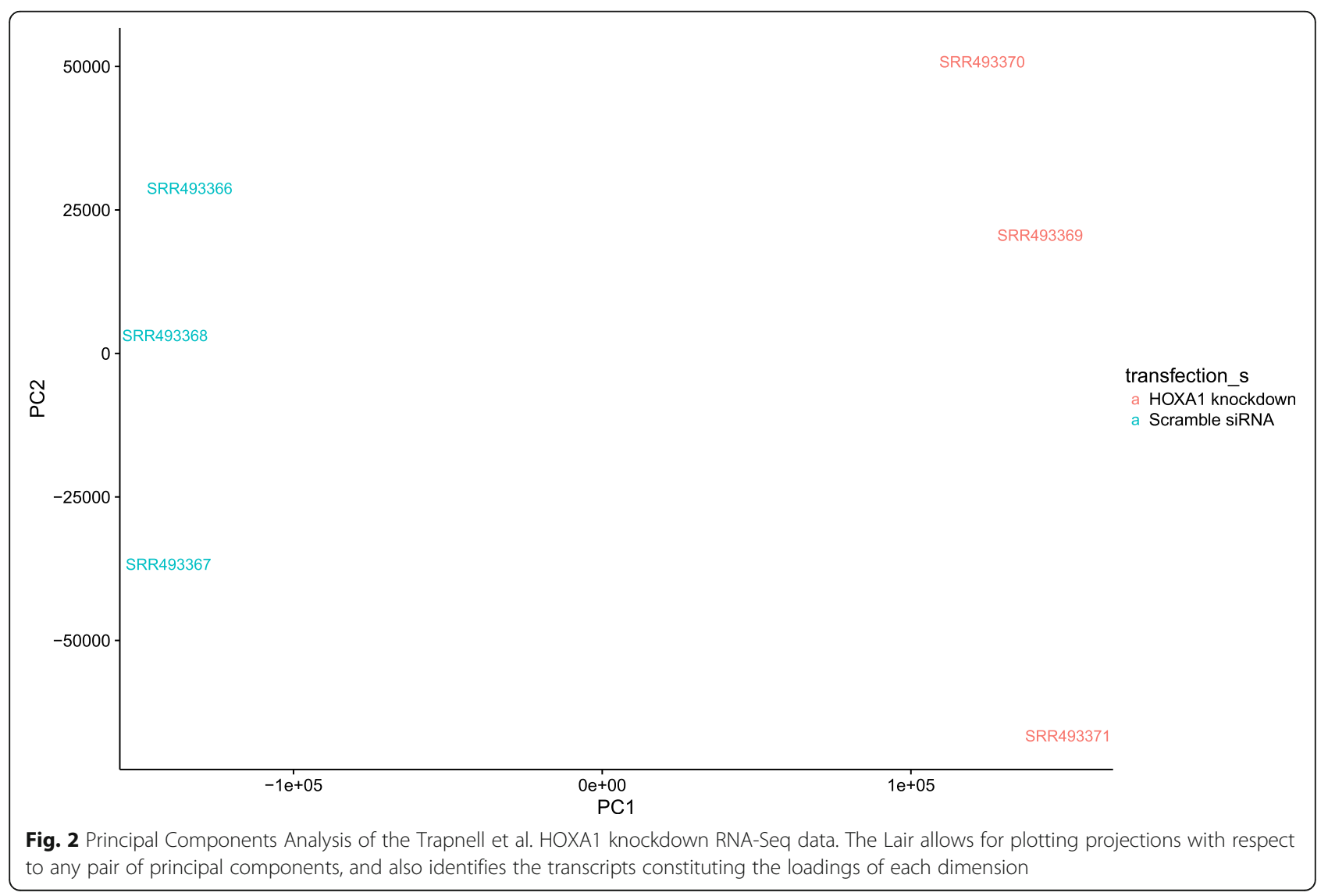


In that paper, an RNA-Seq differential analysis was performed on lung fibroblasts responding to the knockout of the developmental transcription factor HOXA1. First, it is easy to confirm that The Lair analysis replicates the main results of the paper. Figure 2 shows a principal component analysis of the data, confirming high quality data with substantial separation between the two conditions.

Specific results about individual genes that are discussed in the Trapnell et al. paper are easily confirmed. For example, Figure 5 in the Trapnell et al. paper shows transcript abundance changes in response to the knockdown, providing examples of some key genes of interest. The T-box DNA binding domain TBX3 displays an increase in exactly one out of three isoforms (Figure $5 \mathrm{~d}$ in Trapnell et al.). The differential isoform, ENST00000349155, is displayed via the "transcript view" feature in the Shiny app as shown in Fig. 3. The associated q-value in the sleuth test is $\mathrm{q}=3.15 \mathrm{e}-05$. The two remaining isoforms of the gene are not significantly differential, in concordance with the Trapnell et al. paper (for ENST00000257566, q $=0.148$ and in the case of ENST00000613550, the isoform did not pass the requisite filters to be tested).

While the reproducibility of results is reassuring, the innovation in The Lair resource is the ability to go beyond the limited view of the data provided by the authors. In the Trapnell et al. example there are thousands of significantly differential transcripts, and The Lair allows for viewing the raw quantifications underlying each of them. Another advantage is the ability to examine results of different papers analyzed with the same framework. For example, the Ng et al. [15] paper is immediately established to have much higher variance in the estimates due to having fewer replicates in each condition (two instead of three), and the common framework underlying its analysis provides a quantification of that assessment.

\section{Conclusions}

RNA-Seq technology provides rich and complex data for analysis in projects where expression dynamics are of interest. While investigators are eager to squeeze every bit of information out of their data, there are a number of reasons why they are unlikely to be able to do so at the time of publication of their work: analysis methods and tools improve over time and data may be revealed to be useful for applications not considered at the time of acquisition.

The Lair resource we have developed opens up large volumes of RNA-Seq data for both general and targeted

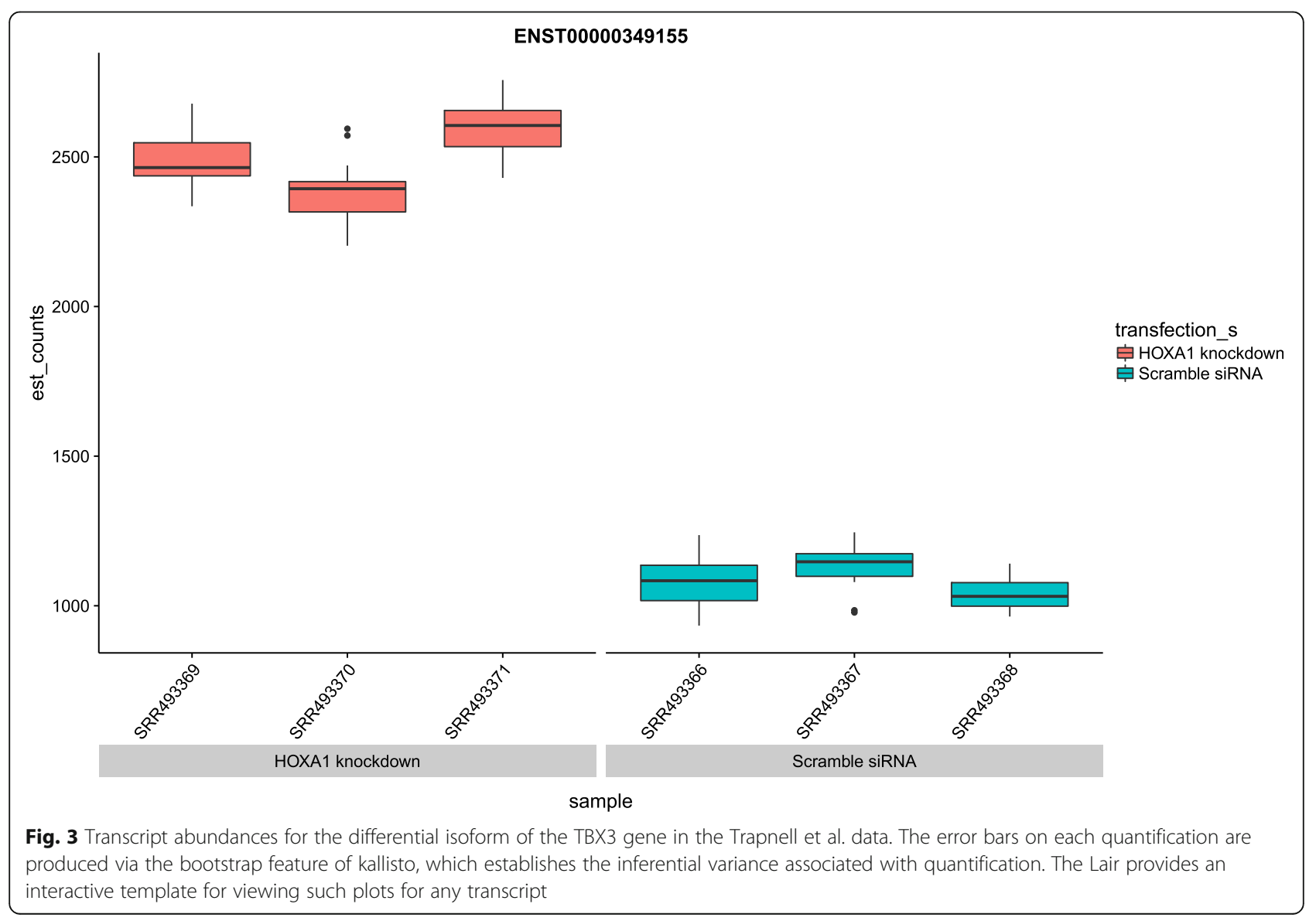


exploration. The modular and automated construction of our system will allow us to upgrade it over time, adding functionality and analyses as we improve and expand the kallisto and sleuth methods and programs. An added benefit of our holistic analysis of SRA data is that our use of the same tools to process diverse datasets also allows for comparison of results across studies. Future plans for The Lair include facilitating such cross-study comparisons.

While we have focused The Lair on bulk RNA-Seq data, the ideas and tools developed in this work should be adaptable to other data types, such as single-cell RNA-Seq, ChIP-Seq, and other high-throughput sequencing experiments. Hopefully such work will establish tools that create symbiotic rather than parasitic relationships between data generators and data analyzers.

\section{Abbreviations}

EDA: exploratory data analysis; GEO: Gene Expression Omnibus;

SRA: sequence read archive

\section{Acknowledgements}

We thank the members of the Pachter Lab for contributing design and feature ideas and for suggesting the initial datasets with which to prototype The Lair. We thank the reviewers for testing our software and providing helpful comments and suggestions.

\section{Funding}

HP and LP were partially supported by NIH grants R01 HG006129, R01 DK094699 and R01 HG008164.

\section{Availability of data and materials}

The main user website and database is at http://pachterlab.github.io/lair. The workflow software is at https://github.com/pachterlab/bears_analyses and the website code is at http://pachterlab.github.io/lair. Access to all webpages is free of charge. All software is released under GPLv3.

\section{Authors' contributions}

HP, NB, PM and LP conceived the idea. HP and PS developed The Lair system. HP, PS and LP analyzed data. HP and LP wrote the manuscript. All authors read and approved the final manuscript.

\section{Authors' information}

Not applicable.

\section{Competing interest}

The authors declare that they have no competing interests.

\section{Consent for publication}

Not applicable.

\section{Ethics approval and consent to participate}

Not applicable.

\section{Author details \\ 'Department of Computer Science, University of California, Berkeley, 387 Soda Hall, Berkeley 94720, USA. ${ }^{2}$ Department of Computer Science, University of Michigan, 486 Vassar Avenue, Berkeley, CA 94708, USA. ${ }^{3}$ Innovative Genomics Initiative, University of California, Berkeley, 188 Li Ka Shing Center, Berkeley, CA 94720, USA. ${ }^{4}$ Faculty of Industrial Engineering, Mechanical Engineering and Computer Science, University of Iceland, Dunhagi 5, 107 Reykjavík, Iceland. ${ }^{5}$ Departments of Mathematics and Molecular \& Cell Biology, University of California at Berkeley, Berkeley, CA 94720-3840, USA.}

Received: 3 June 2016 Accepted: 19 November 2016 Published online: 01 December 2016

\section{References}

1. Frazee $A C$, Langmead B, Leek JT. ReCount: A multi-experiment resource of analysis-ready RNA-seq gene count datasets. BMC Bioinforma. 2011;12:449.

2. Nellore A, Collado-Torres L, Jaffe AE, Alquicira-Hernández J, Pritt J, Morton J, et al. Rail-RNA: scalable analysis of RNA-seq splicing and coverage. Bioinformatics. 2016.

3. Stodden V, Leisch F, Peng RD. Implementing Reproducible Research. Boca Raton: CRC Press; 2014.

4. Conesa A, Madrigal P, Tarazona S, Gomez-Cabrero D, Cervera A, McPherson A, et al. A survey of best practices for RNA-seq data analysis. Genome Biol. 2016;17:13.

5. Bhuvaneshwar K, Sulakhe D, Gauba R, Rodriguez A, Madduri R, Dave U, et al. A case study for cloud based high throughput analysis of NGS data using the globus genomics system. Comput Struct Biotechnol J. 2015;13:64-74.

6. Bray NL, Pimentel H, Melsted P, Pachter L. Near-optimal probabilistic RNA-seq quantification. Nat Biotechnol. 2016;34:525-7.

7. Pimentel H, Bray N, Puente S, Melsted P, Pachter L. sleuth: inspect your RNA-Seq [Internet]. Available from: http://pachterlab.github.io/sleuth/. Accessed 31 May 2016.

8. Chang W, Cheng J, Allaire JJ, Xie Y, McPherson J, RStudio, et al. shiny: Web Application Framework for R [Internet]. 2016 Available from: https://cran.rproject.org/web/packages/shiny/index.html. Accessed 31 May 2016.

9. Köster J, Rahmann S. Snakemake-a scalable bioinformatics workflow engine. Bioinformatics. 2012;28:2520-2.

10. Trapnell C, Hendrickson DG, Sauvageau M, Goff L, Rinn JL, Pachter L. Differential analysis of gene regulation at transcript resolution with RNA-seq. Nat Biotechnol. 2013;31:46-53.

11. Flicek P, Amode MR, Barrell D, Beal K, Billis K, Brent S, et al. Ensembl 2014. Nucleic Acids Res. 2014;42:D749-55.

12. Preston-Werner T. Jekyll [Internet]. 2016. Available from: https://jekyllrb.com/. Accessed 31 May 2016.

13. Otto M, Thornton J, Rebert C, Thilo J, XhmikosR, Fenkart H, et al. Bootstrap [Internet]. 2016. Available from: http.//getbootstrap.com/. Accessed 31 May 2016.

14. SpryMedia. DataTables [Internet]. 2016. Available from: https://datatables. net/. Accessed 31 May 2016

15. Ng S-Y, Soh BS, Rodriguez-Muela N, Hendrickson DG, Price F, Rinn JL, et al. Genome-wide RNA-Seq of Human Motor Neurons Implicates Selective ER Stress Activation in Spinal Muscular Atrophy. Cell Stem Cell. 2015;17:569-84.

Submit your next manuscript to BioMed Central and we will help you at every step:

- We accept pre-submission inquiries

- Our selector tool helps you to find the most relevant journal

- We provide round the clock customer support

- Convenient online submission

- Thorough peer review

- Inclusion in PubMed and all major indexing services

- Maximum visibility for your research 